\title{
Autopoiesis, autoorganización y cierre operativo en las organizaciones desde la perspectiva postestructuralista
}

\author{
Josep Pont Vidal \\ Universidade Federal do Pará (UFPA), Brasil \\ josevidal@ufpa.br
}

Resumen: En el marco actual de pluralismo teórico y de búsqueda de soluciones del postestructuralismo filosófico social entre las diversas teorías organizacionales, la teoría general de sistemas sociales de Lubmann ofrece respuestas operativas a distintos puntos de vista y a los sistemas abiertos mediante los conceptos de autorreferencialidad, cierre operativo y autoorganización. Sin embargo, en su abstracción, presenta ambigüedades teóricas que no han sido consideradas en las observaciones empiricas, entre las que sobresalen las controvertidas cuestiones relativas al cierre operativo y a la deshumanización del sujeto.

Palabras clave: Sistemas autorreferenciales, organizaciones, autoorganización y conciencia.

Autopoiesis, self-organization and operative closure in organizations in poststructuralist perspective

Abstract: In the current framework of theoretical pluralism and the search for solutions of philosophical social poststructuralism among various organizational theories, the Niklas Lubmann General Theory of Social Systems provides operational responses to diverse viewpoints and open systems with the concepts of self-referential, close operational and self-organization. However in its abstraction presents theoretical ambiguities that have not been considered in empirical observations, standing controversial issues concerning the operational closure and debumanization of the subject.

Keywords: Autopoietic systems, organizations, self-organization and consciousness. 


\section{La teoría de sistemas autorreferenciales de Luhmann y las teorías organizacionales}

En la segunda década del siglo xxi, las teorías organizacionales y los management and organizational studies (MOS) se encuentran inmersos en un pluralismo teórico que se extiende a la sociología de las organizaciones, y que refleja y reproduce la confusión existente sobre el papel de las organizaciones en la sociedad actual. Hace tres décadas, uno de los debates académicos centrales de la teoría organizacional giraba en torno a la siguiente pregunta: ¿Son las organizaciones sistemas funcionalmente racionales, técnicamente limitados, o son personificaciones socialmente construidas, subjetivamente significativas, de la acción individual? (Astley y De Ven, 1983). Esta cuestión ha sido extensamente respondida desde varias perspectivas teóricas en las teorías organizacionales y en la acción individual (Burns y otros, 2014; Holzer, 2011; Walsh y Meyer, 2006; y Hardy, Phillips y Clegg, 2001), mientras que otras siguen estando abiertas a debates y controversias en la teoría sociológica y la teoría organizacional actuales (Seidl y Becker, 2006; y Cooper y Burrell, 1988).

Los rápidos y profundos cambios que han experimentado las sociedades occidentales desde entonces han tenido un impacto decisivo en las organizaciones, de modo que una de las preguntas actuales es la siguiente: ¿Cómo podemos entender las organizaciones cambiantes de hoy en día? (Walsh, Meyer y Schoonhoven, 2006). En las posibles respuestas convergen transversalmente ideas que se vinculan con la subjetividad, a saber, el reconocimiento de la existencia del ser humano, su acción y su contexto sociocultural, que se consideran como fundamentales en la descripción organizacional, así como nuevas formas de redefinición de las organizaciones. Reconocer la subjetividad y la complejidad del ser humano abre un amplio abanico de posibilidades analíticas y conceptuales en otras áreas y campos científicos. Para los objetivos de este estudio, se ha recurrido, aunque con diferentes premisas e hipótesis de Luhmann, principalmente, a los postulados de la descripción de la conciencia en la fenomenología social y, en concreto, a los postulados de Husserl sobre el interaccionismo simbólico y, en cierta forma, también, a la psicología humanista (Moustakas, 1994)ํ. No se profundizará en

\footnotetext{
1 La psicología humanista de Moustakas y de May establecen un vínculo entre lo externo del individuo, como son la apariencia y la realidad, y el pensamiento reflexivo, fundamentado en el sentimiento y la conciencia. La similitud del pensamiento de Moustakas con el pensamiento de Husserl y Schutz es innegable: «Es “yo" quién es la persona que vive en un mundo con otros, solos todavía inseparable de la comunidad de los demás; Yo, que ve y entiende algo, recién, como si fuera la primera vez; y yo, que llega a conocer significados esenciales inherentes a mi experiencia» (Moustakas, 1994: 264).
} 
la descripción del «yo» y en la acción intencional y comprensión de la psicología humanista ${ }^{2}$, puesto que se apartan del objetivo de este artículo.

La teoría de sistemas autorreferenciales o teoría general de sistemas sociales (TGSS) del sociólogo alemán Luhmann es un sofisticado intento teóricoconceptual de responder a estas cuestiones, y se inserta en el contexto de cambio paradigmático y en posteriores avances de la lógica y la metodología sistémica. Luhmann realizó una transición de la teoría de sistemas abiertos a los sistemas autorreferenciales, con lo que fue posible entender cómo un sistema podía mantener sus límites a pesar de su dependencia de reproducción comunicativa con su entorno. Este hecho fue un claro avance para la teoría organizacional, al entender las organizaciones no solo como formas de reacción a los impulsos externos, sino también como entidades con capacidad de autorreproducción de sus estructuras independientemente del entorno. No obstante, este avance trajo consigo un nuevo problema, derivado de la autorreproducción de los sistemas, con consecuencias para la teoría organizacional, que se resumen en las siguientes cuestiones: ¿Cómo se puede observar una organización sin tener en cuenta al ser humano?, ¿qué problemas comporta la utilización práctica del concepto luhmanniano de cierre operativo?

Para responder a estas cuestiones de la TGSS de Luhmann, se analizarán las posibilidades prácticas de aplicación en las observaciones organizacionales derivadas de tres conceptos centrales de la teoría de Luhmann, que se enmarcan en el debate con la filosofía social postestructuralista: autorreferencia (Selbstreferenz), cierre operativo (operativer Geschlossenheit) y autoorganización (Selbstorganization). Las cuestiones planteadas inducen a analizar el problema que se plantea, primero, con la operacionalidad y la utilidad práctica de estos conceptos en las organizaciones $y$, segundo, con la idea transversal de la deshumanización de lo humano (o periferización de lo humano) en su teoría, y las posibilidades de repensarla en un sentido humano. Como consecuencia de este objetivo, se expondrá la propuesta de utilización de la inclusión antropológica del ser humano en el sistema psíquico plateado en la TGSS de Luhmann.

\section{Postestructuralismo y propuestas técnico- metodológicas en los estudios organizacionales}

Antes de entrar en el debate axiomático, es preciso definir de forma sumaria algunos términos que guían este escrito: primero, el término postestructuralismo como marco explicativo filosófico social en el que se constituyen actualmente

$2 \mathrm{Al}$ respecto, véase la definición y la explicación de la expresión de las intenciones del yo para el futuro (Anscombe, 1963). 
las organizaciones; segundo, cómo se entienden y se delimitan las aportaciones sistémicas de Luhmann desde la perspectiva autorreferencial; y tercero, a qué se hace referencia cuando se habla de nuevas perspectivas construccionistas, en concreto de la actor network theory (en adelante, ANT) ${ }^{3}$ y de la self-organization theory, influenciada por la teoría de sistemas luhmanniana.

En cuanto al postestructuralismo, se trata de un término creado para definir un movimiento filosófico surgido entre los años 1960 y 1970, periodo de desilusión y rechazo de los valores tradicionales de la sociedad burguesa y de surgimiento del movimiento feminista, y que se aplica a ámbitos tan diversos como la filosofía, la historia y la literatura. Esta definición posee cierta afinidad con el posmodernismo ${ }^{4}$ en sus aspectos teóricos y pragmáticos, por lo que debe ser analizada con cierta cautela, y con ella se pretende etiquetar a filósofos y a pensadores que la han rechazado y que siempre se han negado a aceptarla (Davis y Maquis, 2005). Es el caso de filósofos como Derrida, que en sus escritos cuestiona el término de deconstrucción y afirma no ser un deconstruccionista; de Lyotard, que esgrimió argumentos similares cuando se le intentó definir como posmoderno; y de Foucault, que rechazó el estructuralismo, el postestructuralismo y el posmodernismo, y llegó a afirmar que sus escritos no eran estructuralistas. Su influencia es controvertida, ya que el postestructuralismo es visto a menudo como un "posicionamiento disidente», como por ejemplo respecto a las ciencias en general y a los valores establecidos (James, 2005).

Pese a estas observaciones, para algunos autores que delimitan este término a una aparente superación o radicalización del estructuralismo, la línea divisoria con el postestructuralismo, a pesar de ser un intento de establecer una línea temporal, sigue siendo arbitraria y, por lo tanto, está sujeta a la subjetividad (Tadajewski, MaClaran y Parsons, 2011). Como posible intento de otorgarle un sentido al postestructuralismo, se vincula a los postulados sociológicos del interaccionismo simbólico (Berger y Luckmann) y con el predominio de las metodologías de investigación cualitativas. Entre estas posibilidades cualitativas, destacan los intentos de restablecer una práctica con un diálogo filosófico de esta corriente con el feminismo (Done y Knowler, 2011: 841-852), con observaciones del management y la gestión crítica (Alvesson y Deetz, 1999), y con la incorporación de la gestión estratégica (Ezzamel y Willmott, 2010: 75-109).

Un campo de aplicación práctica que ha producido numerosos estudios y publicaciones en la administración, la teoría de las organizaciones y la sociología or-

\footnotetext{
3 Otras teorías, como el decision support system (DDS), están influenciadas en mayor o menor medida por la teoría de sistemas luhmanniana.

4 No se entrará en el debate sobre la modernidad o en su posible «proyecto inacabado». Véase Habermas, J. (1990): Die Moderne. Ein unvollendetes Projekt. Philosophisch-politische Aufsätze.
} 
ganizacional, es el punto de vista técnico-metodológico de la ANT y el conjunto de puntos de vista teóricos que forman la self-organization theory $y^{5}$. Estas teorías son puntos de vista teóricos de marcada influencia construccionista que en el caso del decision support system (en adelante, DSS) ha sido aplicado por consultorías en el campo de la toma de decisiones gerenciales de las organizaciones privadas y públicas, mientras que sus raíces epistemológicas se remontan a paradigmas técnicos y científicos, como en el caso de la ANT. En la última década, destacan las observaciones empíricas que asumen un referencial teórico conceptual en una interfaz entre la teoría de sistemas y la ANT y, en menor medida, la DSS. Profundizar en estos enfoques sobrepasa el objetivo del presente ensayo, por lo que únicamente se describirán sumariamente los aspectos teórico-conceptuales de la ANT que pueden convergir y procurar soluciones comunes con la teoría de sistemas de Luhmann y el postestructuralismo social.

Son numerosas las publicaciones que establecen un vínculo teórico y operacional entre la teoría de sistemas autopoiéticos de Luhmann y la ANT (White, 1995, [1992] 2007 y 2008), en especial en el ámbito de lengua alemana (Holzer, 2011; Reiser-Kapeller, 2011). La perspectiva postestructuralista del enfoque de ANT proviene principalmente del filósofo Bruno Latour. Sus críticas y distanciamiento de la teoría de sistemas provienen de una serie de «pequeños problemas», entre los que destaca la diferenciación entre sujetos humanos (entendidos como entidades animadas) y actores no humanos, referidos como entidades inanimadas en la ANT. Para Callon (1986) no existe de antemano un mundo de las «cosas» y un mundo de los hombres, ya que la naturaleza y la sociedad son efectos de la existencia de redes heterogéneas (Callon y Latour 1981). En cuanto a la noción de lo social, se trata de una red heterogénea formada no solo por seres humanos, sino también por no humanos (cosas), en la que las comunicaciones y los objetos son el medio de comunicación. La premisa de que la sociedad está compuesta por comunicaciones evidencia las coincidencias con los postulados sistémicos autorreferenciales de Luhmann.

Entre los nuevos enfoques del análisis de redes fundamentados en tecnologías que se aplican con frecuencia en los management and organizational studies y en la toma de decisiones, destaca el DSS (Chiu, Liang y Turban, 2014). Se trata de un sistema de información basado en cálculos realizados por computadoras o por personas, que tiene como objetivo apoyar la toma de decisiones organizativas. La utilización del DSS no solo se ha enmarcado como un proceso social de adop-

5 Estas teorías pueden ampliarse con la DDS y la actor-aystem-dynamics (ASD), ambas derivadas o influenciadas por la TGSS y que constituyen modelos para la toma de decisiones. La propuesta teórica de la ADS se ha aplicado y desarrollado en los análisis institucionales, organizacionales y sociales, y permite su aplicación a la observación de grupos, básicamente pequeños, con el objetivo de analizar el funcionamiento y la dinámica de sus agentes en las normas, en aspectos culturales y en la interacción. 
ción o proceso técnico de tomas de decisiones organizacional (básicamente en organizaciones económicas), sino que también se ha aplicado en organizaciones públicas.

Por último, como aportaciones sistémicas autorreferenciales, se hará referencia a una serie de trabajos teóricos que, a pesar de sus controversias o cruces con la teoría de sistemas autopoiéticos de Luhmann, asumen como centrales los conceptos de autopoiesis y, en consecuencia, de sistema y acoplamiento estructural. Por lo tanto, resulta difícil establecer o hablar de una continuidad en la teoría de sistemas sociales de Luhmann, como también hacer alusión a un programa de investigación sistémico, por lo que se ha optado por definirlo como un 'proyecto teórico de sistemas autorreferenciales'. En el contexto del pensamiento sistémico, es necesario diferenciar entre la teoría sociopoiética de Luhmann, que ha dejado una fuerte herencia que se manifiesta en diversas corrientes en el ámbito del trabajo y las observaciones de carácter interdisciplinario, y la teoría de sistemas (sistemas abiertos).

Al abordar la TGSS de Luhmann, es necesario diferenciar el periodo al que se hace referencia, ya que es posible establecer al menos dos fases diferenciadas de su teoría. La primera se centra en el análisis funcional-estructuralista y comprende la década de los años sesenta y setenta, siendo considerada por algunos como «una obra de arte inigualable» (Baecker, 2013). La segunda, más tardía, se inicia probablemente con la inclusión del concepto de autopoiesis, asimilado a partir de los escritos de los neurobiólogos Maturana y Valera, que incluye la noción de observador.

\section{Implicaciones sistémicas en los estudios organizacionales}

Los resultados de los debates en los estudios organizacionales, los nuevos conceptos sistémicos y los puntos de vista teóricos construccionistas no han pasado desapercibidos para los estudiosos de la teoría de sistemas, que han dirigido sus análisis a observar las posibilidades de implementación de la system and control theory (Veselý, 2011), o en un plano teórico, a establecer similitudes entre la autopoiesis y la grounded theory, el concepto del self o la introducción de la subjetividad en las observaciones organizacionales (Hardy, Phillips y Clegg, 2001) o las implicaciones de la autorreferencia (Hernes y Bakken, 2003). La perspectiva teórica de los estudios organizacionales y de los management and organizational studies se ha visto enriquecida al asumir nociones y conceptos como la autorreferencialidad, el automantenimiento, la circularidad, la individualidad o el mantenimiento 
de la identidad ${ }^{6}$. Los resultados de estas propuestas se manifiestan en una serie de argumentos que apoyan el proceso paralelo de aprendizaje individual y organizacional en el contexto de crecimiento y cambio de las organizaciones (Mavrinac, 2006). De la introducción de nuevos conceptos se ha derivado también que sean más numerosos los estudios sobre las organizaciones que fijan su atención en el papel central de la acción humana en el «saber cómo hacer las cosas» en la organización (Orlikowski, 2002) y en el conocimiento como capacidad no estática, sino permanente arraigada en las personas y en los actores que la componen.

Las respuestas dadas son heterogéneas y numerosas, y en cada país proceden del desarrollo de centros de investigación superior de seguimiento y análisis de las organizaciones y de núcleos de análisis sistémico, en concreto de seguimiento de la teoría luhmanniana, en los que se procuran respuestas para la comprensión de la complejidad. Algunos estudiosos indican la existencia de nuevas formas organizativas, Dilemmas of New Organizational Forms, basándose en la hipótesis de la existencia de «fluid organizations» (Schreyögg y Sydow, 2010; y Sydow y Schreyögg, 2013). La utilización de conceptos sistémicos en la teoría de las organizaciones se ha enriquecido mediante la observación de sus operaciones internas y sus comunicaciones con su entorno, mientras que se han revisado los conceptos y las nociones de estabilidad, cambio, unidad y pluralidad (la unidad y la multiplicidad), el todo y las partes, la relación entre lo material y lo vital, y los alcances y las limitaciones del concepto de sistema. Los debates teóricos sobre esta nueva orientación sistémica han girado en torno a las observaciones de sistemas reales (real systems), dirigidas a los sistemas naturales en los que se constataron variables dinámicas y estáticas, y a las posibilidades que ofrecen en términos de controlabilidad, accesibilidad y observabilidad, y las observaciones del estado estático. Por otro lado, la teoría organizacional y el conjunto de los management and organizational studies han incorporado nociones e ideas provenientes de la teoría de sistemas autorreferenciales, al tratarse de sistemas organizados que tienen como objetivo ocuparse de problemas o demandas sociales. Las organizaciones se han observado y analizado como sistemas autoorganizados en conexión con los sistemas que las sustentan (Goldspink y Kay, 2010) y como entidades estructuradas con proceso de modelaje dirigidas a la cooperación (Helbing, Yu y Rauhut, 2011: 177-208).

6 Para Kai Paetow (2005), la identidad conforma la unidad del sistema, y en la gestión de las organizaciones se trata de un tipo de management de la contingencia. 


\section{Autorreferencia, cierre operativo y autoorganización en la TGSS}

En varios de sus escritos, Luhmann hace referencia a las organizaciones. Sin embargo, es en Organization und Entscheidung (Organización y decisión, 2010) publicada en 1981 en su obra Soziologische Aufklärung, (Ilustración sociológica), en la que profundiza en los problemas relativos a su estructura, autodescripción y operaciones propias internas. Las organizaciones ya no aparecen como superestructuras o «sistemas sociales»o, desde una perspectiva funcional, como «sistemas funcionalmente racionales». Luhmann les atribuye la posibilidad de autorreferencia en la creación de sus estructuras internas, como «sistemas autopoiéticos que se producen y reproducen a sí mismos por medio de operaciones propias» (Luhmann, 1982:25), en las que las decisiones constituyen su esencia: «organizaciones como un conjunto de decisiones». En la misma línea de pensamiento autorreferencial, hizo hincapié en los aspectos evolutivos y destacó las organizaciones como «sistemas sociales evolutivos».

Las nociones de autorreferencia, cierre operativo y autoorganización son centrales en la teoría luhmanniana. Las aplica en la observación de los procesos que determinan las causas de la existencia o la desaparición de las organizaciones. Antes de describir estos conceptos, es preciso definir de forma sucinta cómo describe Luhmann las organizaciones. En su teoría de sistemas, el fenómeno de las organizaciones se enmarca en la descripción de la diferenciación sistémica. Las sociedades altamente diferenciadas están compuestas por innumerables tipos de organizaciones, de tal modo que son la forma social dominante en cuanto a realización de actividades. Los sistemas sociales corresponden a los sistemas que operan autopoiéticamente basándose en la comunicación. Luhmann los clasifica en tres niveles: sistemas funcionales, sistemas de interacción y sistemas organizacionales. Desde una perspectiva de integración social, estos sistemas asumen funciones especiales de diferente alcance y se manifiestan, principalmente, en los límites de los sistemas parciales sociales con funciones específicas (como los tribunales de justicia, defensorías públicas, asociaciones de padres de alumnos o agrupaciones específcas), que también pueden manifestarse de forma transversal y corresponder a diferentes sistemas del conflicto.

\subsection{Autorreferencia en las organizaciones}

El concepto de autorreferencialidad proviene de la teoría de la comunicación y la semiótica. Designa la forma en la que opera un sistema y la operación como reproducción de la unidad del sistema que posibilita el contacto con el entorno 
(Luhmann, 1984 y 1995). Los conceptos de autorreferencia y autopoiesis implican asumir una serie de estructuras, componentes, procesos y operaciones que están imbricados en una lógica de producción y operación cíclica y recursiva dentro del sistema. Una organización autopoiética puede definirse como una red de producción de componentes que simultáneamente actúan bajo dos condiciones: $a$ ) participar de forma recursiva en la misma red de producción de sus componentes y $b$ ) realizar la red de producciones y operaciones como una unidad en el espacio, en el que, a su vez, existen los componentes. A través de las organizaciones los sistemas sociales funcionales realizan sus operaciones e inician mecanismos de inclusión y exclusión en la sociedad. Para Luhmann y algunos teóricos que forman parte de la denominada escuela sistémica de Bielefeld ${ }^{7}$, las comunicaciones decisionales que tienen lugar en las organizaciones constituyen sus operaciones constitutivas, ya que estas operaciones permiten definir sus objetivos y metas, además de los criterios de pertenencia para sus eventuales miembros y la configuración de sus entornos.

Para Luhmann, autopoiesis hace referencia a todas las operaciones (y estructuras) que tienen lugar en el sistema, mientras que la idea de autorreferencia remite a la formación de estructuras dentro del sistema (comp. Nafarrate, 1991:21). El concepto de autorreferencia se erige en el núcleo de los sistemas al constituirse a través de comunicaciones, y también remite a la forma en la que opera un sistema respecto a su entorno. En este sentido, la hipótesis sistémica autopoiética parte de la premisa de que la acción que tiene lugar en las organizaciones no está previamente determinada por el entorno, sino, inicialmente, por una lógica interna precisa (Schimank, 1985: 13). En la perspectiva de la teoría de sistemas sociales y la idea de autorreferencia, se reconoce que las organizaciones poseen una inteligencia sistémica y, con ello, una gestión del conocimiento organizacional. A partir de esta línea argumentativa se encuentran la organización, entendida como un sistema y constituida por su historia; el sistema de reglas; los procesos de gestión; y las formas de transacciones (Willke, 1998: 31-32).

Para Luhmann, la autorreferencia se sitúa en un plano operativo similar al de la autopoiesis, que designa la particularidad de los sistemas, considerada esta como la ampliación permanente y continua de producción de elementos del sistema mediante elementos del propio sistema «cuando el mismo constituye los elementos» (Luhmann, 1984: 16) que le dan forma. Los sistemas autorreferenciales operan necesariamente a partir del «autocontacto», además de ser cerrados y al mismo tiempo, abiertos. El cierre se realiza por medio de la circularidad reflexiva, es decir, de la capacidad estructuralmente asegurada de los elementos para la autorreproducción.

7 Formada por Luhmann, Tyrell, Japp, Willke y Schimank, entre otros. 
Las operaciones sistémicas de autorregulación, autorreferencialidad y autoorganización son requisitos necesarios para la creación de sistemas autónomos, además de ser las condiciones para la supervivencia de las organizaciones. La autorreferencia de los sistemas es la condición para lograr su evolución. Aunque reconoce que las personas «no pueden existir» sin los sistemas sociales, estas ya no forman parte de dichos sistemas, sino que se encuentran situadas en el entorno. En esta tesis, aparecen cuestiones, poco desarrolladas por Luhmann, relativas al reconocimiento de la existencia a priori de sistemas sociales (Tyrell, 1998).

La autorreferencia consiste en la operación mediante la cual el sistema remite sus operaciones a sí mismo (su estructura), lo que permite observar su entorno y, a su vez, realizar distinciones para seleccionar lo que no está en su capacidad estructural. La operación de autopoiesis u organización del sistema como forma para la autorreproducción interna permanente le permite prescindir de los impulsos provenientes del entorno. Al operar de forma autorreferencial, el sistema puede tomar dos alternativas: la primera consiste en asumir diversas funciones con su respectiva estructuración y realizar propuestas de políticas independientemente del entorno; y la segunda, en observar el entorno independientemente de las acciones estructurantes que se lleven a cabo, puesto que se manifestarán en acciones y propuestas cuya efectividad podrá ser analizada empíricamente. En las organizaciones públicas, se trata de un sistema que permite observar las premisas teóricas expuestas, al constituir sus propias estructuras independientemente del entorno, y simultáneamente, observa el entorno para comprobar la efectividad de distintas medidas, entre las que destaca la burocracia como una de las operaciones clásicas del sistema administrativo. La autorreferencia debe estar vinculada a la referencia externa, puesto que la comunicación como observación debe estar dirigida a algo y previamente debe estar tematizada sobre lo que comunica. De este modo, los sistemas sociales, a través de su cierre autorreferencial, pueden crear también la apertura necesaria. Los sistemas de comunicación son sistemas operativamente cerrados (es decir, autorreferenciados) y, al mismo tiempo, como referencias externas, remiten mediante sus operaciones a otros sistemas. Con estas operaciones se descarta que la comunicación no comunique pueda comunicar con, y sobre el entorno, puesto que el mismo sistema social comunica a través de la autoobservación. 


\subsection{Autoorganización}

La idea de autoorganización aparece en la teoría de sistemas y forma parte de una teoría específica, la teoría de la autoorganización ${ }^{8}$. En el campo organizacional, en la última década han aparecido numerosas publicaciones que se centran en estudios de caso con aplicaciones en los aspectos cognitivos de las organizaciones (Van Orden, Holden y Turvey, 2003), siendo incluso calificado de «attractive chance for the development of new ideas in management theory and practice» (Klimecki, 1995: 1). El concepto de autoorganización aporta un vínculo entre la dinámica estructural representada por un modelo de sistema complejo, en el que se tienen en cuenta las condiciones iniciales y del entorno, y la emergencia de nuevas funciones observables en el sistema modelado, siempre y cuando estas funciones no hayan sido inducidas por agentes externos al sistema, es decir, que necesariamente hayan sido engendradas por el propio sistema. En la teoría sistémica luhmanniana, se ha tratado el concepto de autoorganización de forma más bien secundaria y como medio para una operación autopoiética. Luhmann parte de la premisa de que un sistema autopoiético puede variar sus propias estructuras internas dando lugar a una operación autoorganizativa, siempre que esta sea compatible con la operación autopoiética, es decir, que toda la operación incluya también las estructuras. El sistema siempre se encuentra en un estado de incertidumbre sobre sí mismo en relación con el entorno, mientras que la autoorganización se encarga de controlarla y producirla.

Una organización es un sistema que se «autoorganiza, autoconduce y autodetermina» (Jara-Díez, 2006), por lo que la autoorganización está presente en todo tipo de organizaciones, en especial en las económicas, una idea que es considerada como un nuevo paradigma en las ciencias del management. Las organizaciones son sistemas dinámicos y no lineales en los que convergen la estabilidad y la inestabilidad, la evolución y la revolución y, en consecuencia, son entidades caóticas por naturaleza. También son múltiples los actores que componen la organización y que influyen en ella de forma dinámica. De acuerdo con estas premisas, es posible concebir las organizaciones como sistemas «dinámicos no lineales» (Thiétart y Forgues, 1993), de lo que se deriva que el comportamiento organizacional es casi impredecible, sobre todo a largo plazo. Diferente es la distinción a pequeña escala y a corto plazo pudiéndose predecir su comportamiento, al tratarse en este caso de «sistemas disipativos» que cambian continuamente recursos e informaciones con su ambiente o entorno. El conjunto de premisas expuestas lleva a afir-

8 En la tradición histórica del movimiento obrero se ha puesto en práctica la autoorganización económica en diversos países europeos, con una fuerte influencia del movimiento libertario y anarcosindicalista, y con experiencias históricas concretas. 
mar que las organizaciones son, en cierta medida, «generadoras de caos», que a su vez, favorece y sirve para una mejor adecuación de la organización a las diversas demandas de su entorno.

En la comprensión de una organización como sistema dinámico, la capacidad para autoorganizarse es innata en todas las organizaciones y puede ser entendida como un proceso de emergencia de «atractores», es decir, de conjuntos o de trayectorias con las que un sistema evoluciona con el tiempo y que favorecen la autoorganización. Mientras que las organizaciones económicas están sujetas a los problemas derivados de esta incertidumbre, no sucede lo mismo en la Administración pública, puesto que está sujeta a rígidos determinantes normativos y políticos, aunque no por ello está exenta de los impactos procedentes de su entorno, como son las necesarias adaptaciones y reestructuraciones reclamadas por la ciudadanía (Ramió, 2012). En las organizaciones públicas, y a pesar de los principios normativos jurídicamente establecidos, los procesos de autoorganización se estructuran en diferentes niveles, que pueden manifestarse en el paso de los modelos burocráticos al modelo gerencial, o de las propias transformaciones de la Administración pública en diversos ámbitos, como son las instituciones de enseñanza superior (IES), las entidades supramunicipales, las autarquías, las agencias estatales, las empresas mixtas o las corporaciones estatales ${ }^{9}$. En España, las comunidades autónomas son un ejemplo de autoorganización administrativa y de gestión descentralizada del poder público. La autoorganización y los procesos de cambio y de inestabilidad que la acompañan, con los consecuentes desequilibrios del sistema, pueden ser entendidos también como un proceso de bifurcación, que se manifiesta en condiciones alejadas del equilibrio. Estas condiciones producen cambios en la organización y pueden llevar al sistema a un estado de inestabilidad o de cambio mediante eventos pequeños y aleatorios, del que emergen, en consecuencia, nuevas estructuras. El pensamiento sistémico (y las teorías del caos) aplicado a las organizaciones muestra que no es posible ignorar el caos al ser este intrínseco a las organizaciones. Es preferible aceptarlo y dejarlo fluir, y asumirlo como un proceso de aprendizaje para aprovecharlo con vistas a la transformación de la organización, lo que resulta un medio fértil para nuevas metas.

La idea de autoorganización no significa lo mismo que las ideas de autogestión y de autonomía ${ }^{10}$. Mientras que la autogestión está dirigida a la gestión de la organización, la autonomía viene a significar la capacidad para actuar según las

9 Un ejemplo en América latina de entidad estatal con capacidad de autoorganización, lo constituye la Corporación de Fomento a la Producción (CORFO) en Chile, que tiene entre otros objetivos la implementación de políticas públicas para el estímulo de la producción.

10 En el ámbito político, la autonomía implica autoorganización y se vincula con las instituciones de autogobierno y las instituciones autónomas propias, así como con la potestad de autoorganización sobre las instituciones de autogobierno y el régimen estatutario de sus funcionarios públicos. 
propias normas, contraponiéndose a la idea de heteromía (Castoriadis, [1975] 2007). En las organizaciones económicas, pueden pervivir simultáneamente las ideas de autoorganización y autonomía basándose en un equilibrio entre ambas, como muestran varios estudios, en especial en el área de la gestión de la salud y de la gestión hospitalaria. Un ejemplo del difícil equilibrio en la práctica de la autonomía en la prestación de servicios lo encontramos en el Instituto Catalán de la Salud y en la relación jurídica y económica con los consorcios sanitarios. En el ámbito sanitario se ha planteado el dilema del equilibrio entre la autogestión y la autonomía, que se ilustra en el proceso empírico experimental de aumentar la autonomía de los equipos de gestión sanitaria (Informe SESPAS 2012) ${ }^{11}$, Las conclusiones del Informe SESPAS son positivas, puesto que los centros sanitarios autogestionados se sitúan en los primeros puestos de la «banda baja» del gasto farmacéutico en Cataluña. Sin embargo, como resultado se desprende que el recorrido llevado a cabo para la mejora de la autonomía de la gestión «posiblemente esté agotado», cuya causa principal es «el fracaso de modular equipos capaces de autogestionarse sin salir del marco de la gestión pública», además de existir otros riesgos. Los resultados de este informe ponen de manifiesto el problema derivado de las trabas burocráticas, debido a su extrema autorreferencialidad y normatividad burocrática para lograr aplicar procesos de autogestión pública.

\subsection{Cierre operativo (operativer Gescblossenbeit)}

La idea de cierre operativo luhmanniana se refiere a la operación que se produce cuando un sistema logra clausurarse y aislarse de su entorno, pudiendo constituir una realidad diferente, lo que se realiza por medio de las operaciones autorreferencial y autopoiética. No obstante, la noción de cierre operativo ofrece una serie de dificultades operacionales en las organizaciones, puesto que contrapone la necesaria apertura del sistema respecto a su entorno con filtros y limitaciones comunicacionales (Luhmann, 1995). Consciente de las contradicciones que comporta este concepto, Luhmann quiso especificarlo y detallarlo desvinculándolo de la idea de un aislacionismo del sistema respecto a su entorno, ya que esta operación puede inducir a la desaparición del sistema: «no se quiere dar a entender un aislamiento respecto del entorno, ni tampoco el tan divulgado supuesto de una disgregación termodinámica en dirección a la entropía» (Luhmann, 1993: 44). El cierre operativo es un proceso continuo y se compone de los elementos de autoformación y automantenimiento, por lo que necesita una apertura basada en el intercambio de energía y de materia con su entorno. Se trata, pues, de una relación de dependencia y de condiciones que se posibilitan unas a otras. El

11 Castelltort, A. (2012): «¿Autogestión o autonomía de gestión?», Gaceta Sanitaria. 
intercambio entre el sistema y el entorno no está determinado por este último (como sucede en los sistemas abiertos), sino por la determinación de la organización cerrada del sistema autopoiético. Esta operación no significa que el sistema esté completamente y siempre cerrado al entorno, sino al contrario. $\mathrm{Al}$ respecto, Kneer y Nassehi constatan que «el cierre autopoiético de la organización es la condición para su apertura» (Kneer y Nassehi, 1993: 51). Estas ideas nos remiten, en el plano teórico, al significado otorgado por Luhmann a los conceptos de autonomía y autarquía. Para Luhmann, los sistemas autopoiéticos son autónomos, pero no autárquicos, puesto que la entrada y la salida de energía son determinadas por sus propias operaciones sistémicas. En cambio, no son autárquicos, puesto que están supeditados a un entorno concreto en los aspectos materiales y de entrada de energía.

En la operación de cierre operativo, el sistema solo puede operar en sus propias operaciones (estructuras producidas), también denominadas autoorganización o determinismo estructural (Luhmann, 2010: 75). En este sentido, surge la cuestión no resuelta de aclarar y determinar el nivel de autoorganización necesario para hacer posible la autopoiesis de la comunicación entre el sistema y el entorno. En todo sistema aparece la incertidumbre, que puede usarse como recurso autoorganizativo del sistema. En una organización, la autoorganización se extiende en el momento en el que es posible decidir cómo se coordinan las decisiones. Aparecen entonces y son visibles diversas alternativas posibles, lo que se refleja en la racionalidad de las decisiones. La definición de nuevos conceptos y diferenciaciones dependerá del observador, que establecerá la posibilidad de apertura del sistema: «estriba en señalar con qué conceptos, con qué diferenciaciones suplementarias puede exponerse la relación entre el sistema y el entorno» (Luhmann, 1993: 44). A pesar de esta aclaración, la operacionalización de este concepto en observaciones empíricas en organizaciones sigue comportando problemas teóricos relativos a si las organizaciones deben ser entendidas y descritas en el ámbito cognitivo o en el operativo organizacional, puesto que vincularse con uno u otro entendimiento conceptual tiene consecuencias en su observación (Willke, 1998).

En las sociedades contemporáneas, las organizaciones asumen los principios de la autoorganización y, por ello, se encuentran continuamente confrontadas con problemas derivados de la indeterminación del futuro y del riesgo de su entorno. Para minimizar este riesgo, deben reaccionar mediante su capacidad para tomar decisiones de forma imaginativa, por ejemplo, a través de la introducción de la integración vertical de sus procesos de decisión. Esto significa que las decisiones pueden ser iniciadas en todos los niveles de la organización. La autoorganización tiene sentido cuando es capaz de realizar distinciones. Luhmann reconoce que 
es necesaria una «organización de la organización» para que el sistema posea las condiciones necesarias para ofrecerse a sí mismo las posibilidades de decidir sobre las premisas de la decisión, en concreto, de la planificación organizacional (Luhmann, 2010:355). La organización se entiende, pues, como «pura posibilidad», como operaciones de delimitaciones que solo pueden lograrse a través de la autoorganización, es decir, mediante sus propias operaciones.

Aplicar el cierre operativo en un plano teórico-conceptual y la posibilidad de ser asumidos por la organización requieren la necesaria apertura del sistema, ya que en la aplicación práctica de la teoría sistémica de Luhmann surgen cuestionamientos. Ya en la utilización y en la aplicación práctica de este concepto, Willke (1998: 32) cuestiona su uso en las observaciones empíricas: ¿Cómo pueden los sistemas establecer relaciones y cooperar entre sí si sus operaciones se realizan de forma cerrada?, ¿no se adhiere la idea de cierre operativo a una tendencia que se aísla y es ajena al mundo y al entorno? Para Willke, la posibilidad de aplicar el concepto de cierre operativo en las organizaciones aparece cuando los elementos operativos de un sistema (las comunicaciones, las decisiones y las acciones) se entrelazan de forma circular y autorreferencial y, como consecuencia, se establecen formas y procesos específicos de forma circular, de modo que se constituye e intensifica entre ellos una «estructura de redes autocatalítica» (Willke, 1998: 32 33). La consecuencia de esta decisión para la organización es que se constituyen entrelazamientos circulares - en el sentido dado por Manfred Eigen - en un sistema o, en este caso, en una organización, que opera de forma organizacional «hipercíclica».

Asimismo, surgen dudas sobre la adecuada interpretación teórica y la aplicación de este concepto en las observaciones propuestas por Luhmann. Los sistemas autopoiéticos son sistemas cerrados y al mismo tiempo, sistemas abiertos. El cierre se produce mediante una circularidad recursiva, es decir, por medio de su capacidad garantizada de que los elementos operan para la autorreproducción. Estos elementos del sistema no son determinados por el entorno, sino por medio de la cohesión de los sistemas autopoiéticos, de tal forma que no necesitan depender del entorno. No obstante, también están «cognitivamente abiertos»al entorno, puesto que sin este entorno no pueden autorreproducirse, según la idea de diferenciación con el entorno.

Estos presupuestos teóricos son claramente observables en las organizaciones de carácter privado (económicas), de cuya correcta interpretación por parte de su dirección o consejo dependerá su éxito o fracaso y, en última instancia, su desaparición. Este tipo de organizaciones económicas reciben constantemente señales de aviso de su entorno (clientes, cambios en el mercado, proveedores, etc.). El 
modo de reaccionar a estos avisos variará de una empresa a otra. Mientras que unas empresas reaccionan frente a estos avisos externos por medio de decisiones tomadas desde la dirección, en otras empresas son las propias personas que componen la organización (trabajadores y empleados) las que inician, de forma inadvertida y a nivel interno, los cambios necesarios. En este caso, lo importante sigue siendo la lógica operacional del sistema de reglas anónimas existentes y la identificación colectiva de los trabajadores y empleados con los objetivos de la empresa. Para la observación y el análisis científicos y teóricos, los principales problemas de asumir esta lógica se sitúan en torno a la pregunta: ¿Cómo pueden observarse simultáneamente el saber organizacional, sea en las organizaciones públicas o privadas, y las personas? La pregunta presupone la existencia de personas, es decir, la existencia de la subjetividad, como condición previa a la diferenciación del sistema.

En observaciones empíricas, Willke ya pone de manifiesto que la existencia de la autorreferencia y el cierre operativo pueden actuar de forma destructiva y «patológica» en el aprendizaje organizacional. Asumir estas dos ideas luhmannianas (autorreferencialidad y cierre operativo) de forma absoluta y dogmática en las organizaciones puede conducir a que la organización económica se destruya, ya que no tener en cuenta o restringir las señales procedentes del entorno puede propiciar su desaparición. Incluso puede suceder que la organización reaccione en los niveles internos, como es el elemento humano o gerencial, mientras predomine la lógica de organización insensible o congelada a los inputs, comunicaciones o energía precedentes del entorno. Por otro lado, aparecen también descripciones de operaciones realizadas en organizaciones en las que el trabajo del conocimiento de los trabajadores empleados funciona porque las bases del conocimiento organizacional pueden ser entendidas en diferentes realidades. Entender que el conocimiento no es una capacidad estática de la empresa, sino un logro social permanente y reconstituido por actores (sujetos humanos) es un requisito necesario en las observaciones sistémicas, que de forma permanente ha sido ya constatado en observaciones sobre su incidencia en las organizaciones (Orlikowski, 2002). Los resultados de esta constatación tienen como consecuencia que la calidad de los productos o de las comunicaciones con el entorno que produce la organización dependan de su conocimiento profundo del entorno, de estructuras muy arraigadas y de las condiciones sociales del contexto en el que se desarrolla su ámbito económico. El cierre operativo, en definitiva, permite un ejercicio teórico y abstracto sobre la lógica y los nexos y las conexiones comunicacionales y circulares de una organización. Sin embargo, en su aplicación práctica es necesario reforzar y profundizar sus aspectos cognitivos, que son las 
comunicaciones, que abren un amplio espacio de observación al incluir elementos emocionales, culturales, simbólicos, psicológicos y del medio ambiente o de las operaciones del propio sistema.

\section{La inclusión del ser humano como superación de las limitaciones autorreferenciales}

En las observaciones organizacionales, es una cuestión central asumir la existencia de lo humano, con lo que se reconoce la participación activa, en sus diferentes facetas, de las personas que las componen, como ponen de manifiesto distintas observaciones empíricas, ya sea en el nivel más bajo como en los niveles de gerencia y dirección (Willke, 1998 y 1999). El objetivo de este ejercicio teórico ha sido proponer la idea de una relación entre sistema psíquico y conciencia, aspectos que en la TGSS de Luhmann han sido fuente de cuestionamientos en los planos ontológico y antropológico. El objetivo ha sido superar las controversias teóricas de la teoría luhmanniana mediante la propuesta de reconocimiento de la existencia previa de una conciencia como punto de partida en las operaciones observacionales de los sistemas, en los que la comunicación, no solo en forma de energías (inputs) o simbólica, sino también lingüística, se establece como nexo comunicacional entre los sistemas. Siguiendo esta línea argumentativa conceptual, también se cuestiona la premisa central de la ANT, al externalizar lo humano de los actores que componen una red. Las consecuencias de asumir estas proposiciones implican repensar las semánticas y las operaciones subyacentes que configuran el sistema psíquico y la conciencia en la TGSS.

Luhmann reconoce que sin la existencia de personas no puede haber sociedad, de forma similar que no puede haber comunicación sin conciencia. El comportamiento del individuo (Mensch) «puede ser regulado y suprimido» mediante la conciencia, en la que la comunicación «puede ser utilizada para ampliar la conciencia y asumir determinados temas» (Luhmann 1998: 306). Dos elementos sobresalen en la definición: el papel de la conciencia como desencadenante y la comunicación. Para la descripción de la conciencia, Luhmann busca en las fuentes de la sociología fenomenológica de Husserl, por lo que su descripción sucinta se hace imprescindible. Este filósofo establece como premisa inicial la diferencia entre el «yo de las vivencias» y el «yo empírico», que se delimitan del «yo psíquico puro» en su contenido fenomenológico, lo que a Luhmann le permite delimitar el «yo» y reducirlo a la unidad de la conciencia. La conciencia, o subjetividad trascendental en su temporalidad, se da a partir de dos formas: la materia subjetiva y la realidad al nivel de lo consciente. Para ello, propone la operación de sucesivas 
epochés que posibilitan establecer conclusiones seguras. Estas operaciones también permiten establecer la temporalidad de la acción, operando siempre en el presente como origen y estructura de la conciencia. La idealidad que garantiza la repetibilidad de ideas y, por lo tanto, la vida trascendental, la constituyen el aspecto empírico y la comunicación, que es una de las operaciones de la conciencia. Estas operaciones permiten a Husserl no dudar de la existencia del mundo, aunque advierte que la operación de reducir la realidad mediante epochés tampoco es una garantía para acceder a la realidad.

Luhmann otorga un significado diferente a la conciencia al abandonar la teoría del conocimiento fundamentada en la subjetividad y poner en duda la propia observación como operación en la obtención del conocimiento. Se aleja de las descripciones de la conciencia trascendental de Husserl y de la conciencia psicológica como cualidad del conocimiento o como un fluir dinámico en el sentido dado por Anscombe. En su lugar, coloca el sistema social, que construye su realidad mediante un proceso comunicacional, en el que la comunicación adquiere la «única garantía para la realidad del sistema social» (Luhmann, 1998: 397). Esta operación permite controlar si la comunicación se ha realizado por medio de una operación interna que tiene su origen en el exterior del sistema. También suprime cualquier referencia o definición empírica, o que pueda ser definida como empírica, como es el lenguaje. Esta tesis no está exenta de paradojas, puesto que la conciencia es entendida como un sistema autopoiético y, por lo tanto, que está cerrado a su entorno, significa que está cerrado en sus estructuras y operaciones, aunque, por tratarse de un sistema autopoiético, también está abierto cognitivamente.

En este sentido, se observa que la comunicación de la conciencia sigue siendo un punto negro de la teoría de Luhmann. Hace referencia explícita a la idea de conciencia y admite su existencia, pero de forma ambigua (Luhmann 1984, 1995a, 1995b y 1995c), puesto que en las condiciones de la TGSS de doble contingencia autorreferencial no es posible una comunicación directa entre sistema psíquico y conciencia. Reconoce que la conciencia posee capacidad autorreferencial, es decir, capacidad para autorreproducirse independientemente de su entorno. En escritos tempranos, sostiene que la operación del sistema psíquico en la conciencia equivale a las representaciones denominadas unidades elementales de conciencia, definidas también como selecciones, que permiten que el sistema psíquico realice operaciones de observación de la comunicación y de la participación en ella. No obstante, en la descripción de la posibilidad de que el sistema psíquico asuma una conciencia, en diversos escritos ${ }^{12}$, utiliza de forma indistinta los con-

12 Luhmann, N. (1985): Die Autopoiesis des Bewusstsein. 
ceptos de sistema psíquico y sistema de conciencia, con un significado y operaciones similares, sin una delimitación clara entre uno y otro significado, puesto que el sistema psíquico realiza las operaciones de observar y participar en la comunicación.

Como sistema, la conciencia puede distinguir las operaciones básicas de autorreferencia (los pensamientos) y de heterorreferencia (las representaciones) como los elementos últimos del sistema psíquico. Estos operan con sentido y selecciones, por lo que se trata de los pensamientos. Los pensamientos como operaciones autorreferenciales poseen también las cualidades de autoobservarse a sí mismos y de observar otros pensamientos, y así permiten que el sistema adopte una limitación adicional, que es la comunicación, de tal forma que es posible deducir que la conciencia participa de la comunicación con estados de conciencia lingüísticamente estructurados. Estas descripciones y los elementos últimos a los que hace referencia Luhmann (1995: 60) pueden ser interpretados como procesos autorreferenciales, es decir, pensamientos que poseen la capacidad para autoobservarse y para observar otros pensamientos. Luhmann recurre a las operaciones derivadas del proceso de comunicación, operaciones que transmiten un sentido, siempre suponiendo que el sistema se «asimetrice a sí mismo» en el acto de comunicar (Luhmann, 1998: 59). El sentido corresponde a la apertura cognitiva de la conciencia a una lógica autorreferencial, atribuyéndole un sentido autorreferencial para reducir la complejidad en la medida que puede prever su propia posibilidad de reactualización.

Para explicar la idea de las representaciones y del sentido, Luhmann recurre de nuevo a la fenomenología trascendental de Husserl, sobre la cual, como se observa, mantiene una posición ambigua de inspiración y de distanciamiento. Para Luhmann, la conciencia asume y opera como una autopoiesis de la propia conciencia, ya que posee la capacidad para producir sus propias estructuras y las consiguientes operaciones dentro del sistema. El cierre operativo que subyace en el sistema autopoiético y la conciencia se reduce a sus operaciones de estructuración del mundo y descripción de los objetos. Con estos argumentos, la conciencia se manifiesta de forma abierta a sus comunicaciones mediante el proceso del lenguaje, operando este como un plus de la conciencia. El lenguaje es el medio por el que la conciencia es autopoiética, ya que permite aumentar la capacidad de sus operaciones. En toda la argumentación luhmanniana siguen apareciendo interrogantes y ambigüedades al definir y concretizar los estados de conciencia, su participación en los pensamientos o la propia noción de conciencia en la comunicación. 


\section{Consideraciones finales: ¿Hacia un construccionismo sistémico?}

En cierto modo, la heterogeneidad y multiplicidad de teorías y conceptos utilizados en los estudios organizacionales convergen en su conjunto hacia una amplia muestra de puntos de vista construccionistas pensados en una lógica sistémica. En los últimos años, el desarrollo del pensamiento científico sistémico sugiere la necesidad de nuevos enfoques para la teoría organizacional y los management and organizational studies en términos de lógicas diferentes a la linealidad tradicional de entender los procesos, la gestión y, en consecuencia, también las observaciones. El paradigma de la TGSS de Luhmann proporciona unos instrumentos conceptuales teóricos y, a su vez, abstractos, que son adecuados para la observación de situaciones de inestabilidad y de riesgo en las organizaciones, aunque el concepto de cierre operativo, en los niveles práctico y empírico, es pensado con cierta cautela, pues puede ser incluso negativo para las organizaciones. Como respuesta a las limitaciones estructurales autopoiéticas del cierre operativo, se propone, en primer lugar, que el concepto se centre en la observación de la autoorganización de la organización, es decir, en la observación de sus estructuras internas autoproducidas; $y$ en segundo lugar, que se profundice en sus aspectos cognitivos, es decir, en las comunicaciones que se establecen con el entorno y que incluyen elementos emocionales, culturales, simbólicos y psicológicos del ser humano y del medio ambiente.

Cabe destacar que la idea que se propone en este estudio sugiere que el punto de partida en la observación no sean los sistemas como entes abstractos, sino que sea el sistema de conciencia, con las atribuciones ontológicas dadas por Husserl. Esta operación permitiría al «frío» postestructuralismo superar los déficits antropológicos colocando a la persona como centro de la TGSS y alejándola de su situación periférica al asumir de nuevo el centro de la acción, aunque con otras perspectivas como punto de partida. De la interacción resultante entre varias personas se forman los sistemas sociales. Con la idea de inclusión de la conciencia, denominada en la TGSS sistema de conciencia, se propone asumir una reconfiguración jerárquica de la configuración propuesta por Luhmann, que incluye las operaciones específicas del sistema psíquico, como son sus conexiones comunicativas. La autorreferencialidad que aparece en estas operaciones tiene lugar en la formación de estructuras en el sistema de conciencia y en sus estructuras mentales, como operaciones internas psíquicas y mentales de cada persona. Las operaciones autorreferenciales del sistema psíquico se realizan con la creación de estructuras derivadas del sentido y la intencionalidad de la conciencia de cada 
persona y que hacen referencia a las operaciones. La existencia de una conciencia es anterior al sistema psíquico, con lo que se abre la posibilidad de la existencia de la capacidad autorreferencial de la conciencia (creación de estructuras propias) y de sus posibilidades de intencionalidad como respuesta a la deshumanización (o periferización del ser humano). Estos argumentos pueden extenderse a los seguidores de la ANT. 


\section{Bibliografía}

Alvesson, M. y Deetz, S. (1999): "Critical theory and postmodernism: approaches to organizational studies», en S. ClegG y C. HARdy (1999): Studying organization: theory and method, Londres: Sage.

Anscombe, G. E. M. (1963): Intention, Harvard University Press. Disponible en <http://www2.warwick.ac.uk/fac/soc/philosophy/undergraduate/modules/ ph351/coursecontent201314/intention-second-edition.pdf >.

Astley, W. G. y De Ven, A. H. (1983): «Central perspectives and debates in organization theory», Administrative Science Quarterly, 28 (2), pp. 245-273.

BAECKER, D. (1991): Womit handeln Banken? Eine Untersuchung zur Risikoverarbeitung in der Wirtschaft. Fráncfort del Meno: Suhrkamp.

- (2002): Wozu Systeme?, Berlín: Kulturverlag Kadmos.

Callon, M.(1986): «Some elements of a sociology of translation: domestication of the scallops and the fischermen of St. Brien Bay», en J. Law (ed.), Action a belief: a new sociology of knowledge?, Londres: Routledge $\&$ Kegan, pp. 196223.

Callon, M. y Latour, B. (1981): «Unscrewing the big Leviathan: how actors macro-structurereality and how sociologists help them to do so», en K. Knorr-Cetina y A. V. Cicourel, Advances in social theory and methodology: toward an integration of micro- and macro-sociologies, BostonLondres: Routledge \& Kegan, pp. 277-303.

Castelltort, A. (2012): «iAutogestión o autonomía de gestión?», Gaceta Sanitaria [en línea], 26 (S), pp. 57-62. <http://www.S0213911111003918_ S300_3s.pdf>.

Castoriadis, C. (2007): La institución imaginaria de la sociedad, Barcelona: Tusquets Editores.

Chiu, C. M.; Liang, T. P., y Turban, E. (2014): «Introduction to the special issue on crowdsourcing and social networks analysis», Decision Support Systems, 65, pp. 1-2.

Cooper, R. y Gibson, B. (1988): «Modernism, postmodernism and organizational analysis: an introduction», Organization Studies, 9 (1), pp. 91-112.

Davis, G. y MaQuis, C. (2005)ः «Prospects for organization theory in the early twenty-first century, international fields a mechanismus», Organization Science, 16 (14), pp. 332-343.

Done, E. J. y Knowler, H. (2011): «(Re)writing reflective practice with Deleuze, Guattari and feminist poststructuralism», Journal: Reflective Practice, 12 (6), pp. 841-852. 
Ezzamel, M. y Willmotт, H. (2010): «Strategy and strategizing; a poststructuralist perspective», en J. A. C. Baum y J. Lampel (eds.), The globalization of strategy research. Advances in strategic management, Emerald Group Publishing Limited, 27, pp. 75-109.

Hardy, C.; Phillips, N., y Clegg, S. (2001): «Reflexivity in organization and management theory: a study of the production of the research "subject" », Human Relations, 54 (5), p. 531.

Hernes, T. y Bakken, T. (2003): «Implications of self-reference: Niklas Luhmann's autopoiesis and organization theory», Organization Studies, 24 (9), pp. 1511-1535.

Holzer, B. (2011): «Die Differenzierung von Netzwerk, Interaktion und Gesellschaft. Erschienen», en M. Bommes y V. Tacke, Netzwerke in der funktional differenzierten Gesellschaft, Wiesbaden: VS Verlag für Sozialwissenschaft, pp. 51-66.

Klimecki, R. (1995): «Self-organization as a new paradigm in management science?», artículo presentado en el 12 EGOS Colloquium, en Estambul, del 6 al 8 julio. Disponible en <http://kops.uni-konstanz.de/bitstream/ handle/123456789/3931/klim10.pdf? sequence $=1>$.

Kneer, G. y Nassemi, A. (1993): Niklas Lubmanns Theorie sozialer Systeme. Eine Einführung, Múnich: UTB Taschenbücher.

Latour, B. (2002): A dialog on actor network theory. Disponible en <http:// www.ensmp.fr/ latour/articles/article/090.html>.

Lummann, N. (1981): «Organization und Entscheidung», Soziologische Aufklärung, Opladen: Westdeutscher Verlag.

- (1982a): Soziologische Auklärung. Beobachtungen der Moderne, Opladen: Westdeutsche Verlag.

- (1982b): «Autopoiesis, Handlung und Kommunikative Verständigung», Zeitschrift für Soziologie, 11, pp. 336-379.

- (1984): Soziale Systeme. Grundrisse einer allgemeinen Theorie, Fráncfort del Meno: Suhrkamp.

- (1993): «Autoorganización e información en el sistema político», Revista de Occidente, 150. p. 44.

- (1995): «Interaktion, Organization, Gesellschaft: Anwendungen der Systemtheorie», Soziologische Aufklärung, Opladen: Westdeutscher Verlag.

Mavrinac, M. A. (2006): «Self as system* comparing the grounded theory of protecting self and autopoiesis», World Futures: The Journal of New Paradigm Research, 62 (7), pp. 516-523.

Mead, H. (1972): Mind, self and society, Chicago: Chicago University Press. 
Moustakas, C. (1994): Phenomenological research, Londres: Sage.

Nafarrate, J. T. (1991): «Nota a la versión en lengua castellana», en $\mathrm{N}$. Luhmann, Sistemas sociales. Lineamientos para una teoría general, Rubí: Anthropos Editorial-Universidad Iberoamericana, pp. 13-25.

Nassehi, A. (2004)ः «Sozialer Sinn», en A. Nassehi y G. NollmanNः Bourdieu und Lubmann. Ein Theorievergleich, Fráncfort del Meno: Suhrkamp.

Nassehi, A. y Nollmann, G. (2004): Bourdieu und Lubmann. Ein Theorienvergleich, Fráncfort del Meno: Suhrkamp.

OrLIKowsKI, W.J. (2002): «Knowing in practice: enacting a collective capability in distributed organizing», Organization Science, Knowledge, Knowing, and Organizations, 13 (3), mayo-junio, pp. 249-273.

Paetow, K. (2005): Organisationsidentität: eine systemtheoretische Analyse der Konstruktion von Identität in der Organisation und ibrer internen wie externen Kommunikation, tesis doctoral, der Philosophie im Fachbereich Sozialwissenschaften der Universität Hamburg.

PeCI, A. (2006)ः «A nova teoria institucional em estudos organizacionais: uma abordagem crítica», Caderno Ebape, iv.

Pérez-Solari, F. y Labraña, J. (2013): «Hacia la observación de una sociedad venidera: una entrevista con Dirk Baecker», Revista Mad, Santiago de Chile, 29, p. 82-91. <www.revistamad.uchile.cl>.

Рокоц, B. (2002): «Contribution to the comparison of the theories of Bourdieu and Luhmann», Jogelméleti Szemle, 3. <http://jesz.ajk.elte.hu/pokol112. html>.

Ramió, C. (2012): «La admistración que se espera para después de la crisis», GIAPP, Estudios Working-Papers, Madrid: Instituto Universitario de Investigación Ortega y Gasset, WP. 2011, pp. 2-14.

Seide, D. y Becker, K. H. (2006): «Organizations as distinction generating and processing systems: Niklas Luhmann's contribution to organization studies», Organization Studies, 13.1, pp. 9-35.

Sydow, J. y Schreyögg, G. (Hrsg.) (2013): Self-reinforcing processes in and among organizations, Londres: Palgrave Macmillan.

Schimank, U. (1987): «Evolution, Selbstreferenz und Steuerung komplexer Organisationssysteme», en M. Glagow y H. Willke: Dezentrale Gesellschaftssteuerung: Probleme der Integration polyzentrischer Gesellschaft, Pfaffenweiler: Centaurus Verlag, pp. 54-64.

Schmidt, S. (1987): «Der Radikale Konstruktivismus: Ein neues Paradigma im interdisziplinären Diskurs», Der Diskurs des Radikalen Konstruktivismus, Fráncfort del Meno: Suhrkamp, pp. 11-88. 
Schreyögg, G. y Sydow, J. (2010)ः «Organizing for fluidity? Dilemmas of new organizational forms», Organization Science, (21), pp. 1251-1262.

Tadajewski, M*; MaClaran, P., y Parsons, E. (2011): Key concepts in critical management studies, Londres: Sage.

Thoenig, J.-C. (2007): «Recuperando a ênfase na dimensão pública dos estudos organizacionais», Revista Administração Pública, edición especial 1967-2007, pp. 9-36.

Tröndle, M. (s.f. $)_{*}$ «Systemtheorie, ein Versuch», <http://www.fachverbandkulturmanagement.org/wp-content/uploads/2012/10/SystemtheorieEinVersuch.pdf>.

Teisman, G.; Van Buuren, A., y Gerrits, L. (eds.) (2009): Managing complex governance systems, Nueva York: Routledge.

Tyrell, H. (1998)ः Religion als Kommunikation, Würzburg* Ergon-Verl.

Van Orden, G*; Holden, J*, y Turvey, M. (2003): «Self-organization of cognitive performance», Journal of Experimental Psychology, 132 (3), pp. $331-351$.

Veselý, K. (2011): «New system theory and its impact on control theory», International Journal of General Systems, 40 (2), pp. 197-216.

Walsh, J. P.; Meyer, A. D., y Schoonhoven, C. B. (2006): «A future for organization theory: living in and living with changing organizations», Organization Science, 17 (5), pp. 657-671.

White, H. C. (1995): «Social Networks Can Resolve Actor Paradoxes in Economics and in Psychology», Journal of Institutional and Theoretical Economics, 151, p. 58-74.

White, H. C. (2007): Identity and Control, Princeton: Princeton University Press.

White, H. C. y Godart, F. (2008): «Linking networks and domains: cultural and discursive formations in context», Manuscript for Manchester talk.

Willke, H. (1998)ः Systemisches Wissensmanagement, Stuttgart: UTB.

- (1999): Systemtheorie II. Interventionstheorie, Stuttgart: UTB, pp.142-156. 